\title{
EDITORIAL \\ Periodical shifts in the surgical correction of sagittal craniosynostosis
}

\author{
Mark M. Souweidane, MD \\ Department of Neurological Surgery and Pediatrics, Weill Cornell Medical College, New York, New York
}

$\mathrm{I}$ T might seem mystifying to the novice practitioner to select the optimal form and timing for treating singlesuture sagittal synostosis. This difficulty is also frustrating to the veteran craniofacial expert, because the surgery du jour seems to transform at a regularity reminiscent of the periodical cicada (17- or 13-year brood). Beginning with the simple suturectomy, to the modified strip craniectomy, to the calvarial vault reconstruction, to the extended vertex craniectomy, to the endoscopic suturectomy with orthotic helmet therapy, it remains uncertain where consensus will ultimately lie. Further complicating this issue is the variance within any of these surgical epochs with minor or substantive modifications all espousing betterment.

In the paper "The incidence of raised intracranial pressure in nonsyndromic sagittal craniosynostosis following primary surgery" in this issue, Dr. Thomas and colleagues present a cautionary note regarding the impact of the surgical technique in delayed elevation of intracranial pressure (ICP) after correction of sagittal synostosis. ${ }^{4}$ As noted in the paper, this uncommon but real phenomenon of raised ICP should already be familiar, but its relationship to the type of corrective procedure is provocative.

Not dissimilar from the incidence reported from other large clinical series (1.5\%-9.0\%), elevated ICP in this cohort of 217 patients was $6.9 \%(n=15) .^{1-3,5}$ The definition of raised ICP was dependent on tangible ICP monitoring. The authors also report that $18 \%(\mathrm{n}=39)$ actually had presented with signs or symptoms suggestive of elevated pressure. This discordance between the clinical spectrum and the actual ICP raises unease with respect to the true incidence and the optimal detection of nonphysiological pressures. In fact, the authors found that "Although all patients who had ICP monitoring presented with new symptoms or signs indicative of raised ICP, no particular symptom or clinical sign was more closely correlated with the presence of raised ICP on subsequent monitoring... Similarly, although patients were usually required to have both positive clinical and radiological findings before undergoing ICP monitoring, no 1 radiological sign correlated better with a later finding of raised ICP than any other." More concerning is that only 1 patient actually had papilledema on funduscopic examination, only $7 \%$ of those defined as having raised ICP by monitoring. This information presented in the paper highlights the fallibility of clinical indicators of elevated ICP in these children but reinforces the need for long-term surveillance and vigilance. The results also suggest a potential need to lower the threshold in performing diagnostic monitoring in this population.

That a modified strip craniectomy (MSC) was more frequently associated with eventual elevated ICP compared with those children undergoing a cranial vault remodeling (hazard ratio [HR] 6.1,95\% CI 1.4-27.1) might or might not be of concern. The true impact of this statement is dependent upon the actual definition of the corrective procedure. One's definition of an MSC might blend imperceptibly with other variations on this theme. The inclusion of radial osteotomies and wedge osteotomies to supplement a strip suturectomy were a welcome evolution in the correction of sagittal synostosis. Currently, simple suturectomy (without orthotic helmet therapy) is not accepted as a legitimate surgical approach in children with scaphocephaly. The modification in MSC is defined here as, "with or without the addition of lateral barrel staving." This difference is important. On one hand, without barrel stave osteotomies, one might predict a poorer outcome, a finding reminiscent of an earlier epoch. On the other hand, if all patients with an MSC who developed raised ICP had radial osteotomies, then there is greater concern 
based on the current results. Similarly, the technique of performing radial osteotomies can vary widely, ranging from simple osteotomies using Tessier scissors, to the same using a high-speed air drill, to performing wedge craniectomies, with the width and radial dimension all having some degree of importance. In summary, that the surgical procedure is related to the potential for secondary cranial restriction is not surprising. What needs to be known, however, is what type of MSC accounted for the majority of children who eventually were found to have ICP elevation. In short, based on the available information in this paper, it would be premature to abandon the technique of strip craniectomy with radial osteotomies in the treatment of nonsyndromic sagittal craniosynostosis.

It would be difficult to ignore the findings of this paper as they might relate to the evolving surgical trend of endoscopic-assisted (minimally invasive) suturectomy with orthotic helmet therapy. This latest "advance" in singlesuture synostosis correction integrates two features that in the current paper led to a significantly greater tendency to develop raised ICP: less bone removal/contouring, and a younger age at the time of correction. These associations mandate rigorous reporting of results with suturectomy and helmet therapy that include meaningful durations of follow-up (the 15 children in this series were diagnosed with elevated ICP at a mean interval of 51 months) and clinical correlatives of raised ICP (symptomatic headaches, developmental quotients, funduscopy, and longitudinal anthropometry).

On initial reading of this paper it is understandable that one might invoke changes to his or her operative approach in treating single-suture sagittal synostosis. On the surface, using a total vault remodeling and treating at an age $>12$ months might appear logical. A clearer understanding of what surgical procedure was associated with an elevated ICP, however, is needed. Yet the confirmatory message cannot be ignored: the correction of sagittal synostosis mandates a long-term comprehensive longitudinal follow-up, even if the components of that surveillance are not clear.

http://thejns.org/doi/abs/10.3171/2014.9.PEDS14350

\section{References}

1. Adamo MA, Pollack IF: A single-center experience with symptomatic postoperative calvarial growth restriction after extended strip craniectomy for sagittal craniosynostosis. J Neurosurg Pediatr 5:131-135, 2010

2. Bonfield CM, Lee PS, Adamo MA, Pollack IF: Surgical treatment of sagittal synostosis by extended strip craniectomy: cranial index, nasofrontal angle, reoperation rate, and a review of the literature. J Craniomaxillofac Surg [epub ahead of print], 2014

3. Marucci DD, Johnston CP, Anslow P, Jayamohan J, Richards PG, Wilkie AO, et al: Implications of a vertex bulge following modified strip craniectomy for sagittal synostosis. Plast Reconstr Surg 122:217-224, 2008

4. Thomas GPL, Johnson D, Byren JC, Judge AD, Jayamohan J, Magdum SA, et al: The incidence of raised intracranial pressure in nonsyndromic sagittal craniosynostosis following primary surgery. J Neurosurg Pediatr [epub ahead of print January 9, 2015. DOI: 10.3171/2014.11.PEDS1426]

5. van Veelen ML, Eelkman Rooda OH, de Jong T, Dammers
R, van Adrichem LN, et al: Results of early surgery for sagittal suture synostosis: long-term follow-up and the occurrence of raised intracranial pressure. Childs Nerv Syst 29:9971005,2003

\section{Response}

\author{
Gregory P. L. Thomas, PhD, FRCS, ${ }^{1}$ \\ David Johnson, DM, FRCS, ${ }^{1}$ Jo C. Byren, MRCGP, 1 \\ Andrew D. Judge, PhD, ${ }^{2,3}$ Jayaratnam Jayamohan, FRCS, ${ }^{1}$ \\ Shailendra A. Magdum, FRCS, ${ }^{1}$ Peter G. Richards, FRCS, ${ }^{1}$ \\ and Steven A. Wall, FCS(SA) Plast ${ }^{1}$
}

${ }^{1}$ Oxford Craniofacial Unit, Oxford University Hospitals NHS Trust, Oxford; 2Oxford NIHR Musculoskeletal Biomedical Research Unit, Nuffield Department of Orthopaedics, Rheumatology and Musculoskeletal Sciences, University of Oxford; and ${ }^{3} \mathrm{MRC}$ Lifecourse Epidemiology Unit, University of Southampton, Southampton General Hospital, Southampton, United Kingdom

We appreciate Dr. Souweidane's considered and balanced editorial regarding our paper. We certainly agree with and wish to emphasize the conclusions he draws regarding the relative fallibility of individual clinical signs in predicting abnormal ICP and hence the need for vigilant, structured, long-term surveillance with a lowered threshold for performing diagnostic monitoring in these patients.

In his editorial, Dr. Souweidane questioned whether the extent of the surgical intervention within the MSC group might relate to the subsequent incidence of raised ICP. Specifically, were rates of raised ICP higher in those patients who had not undergone lateral barrel staving (lateral radial osteotomies) as part of their primary procedure, when compared with those who did? This was not a question we had addressed in the paper. However, of the 13 children who proved to have elevated ICP following MSC, 8 (61.5\%) had undergone lateral barrel staving, with no difference in the incidence of postsurgical raised ICP between these patients and those individuals who had only undergone a wide-vertex sagittal suturectomy.

The use of a wide-vertex sagittal suturectomy of a minimum 5-cm width is a modification of the narrow 2to $3-\mathrm{cm}$ linear suturectomies of an earlier era. The addition of lateral barrel-staving to the wide-vertex sagittal suturectomy was performed more frequently as the series progressed, being first employed in 1998, in two-thirds of MSC cases between 2000 and 2004, and finally in more than $80 \%$ from 2005 . The technique was not rigidly standardized, but rather the number and extent of radial osteotomies that were made varied both between surgeons and over time, although Fig. 1 in our paper is representative of the majority of these procedures. We did not attempt to quantify the extent of lateral barrel staving within the MSC group, because the available retrospective data, in the form of written operative notes, was not always of sufficient detail. Because of these potential confounding factors we chose not to present this subgroup analysis. However, we are satisfied that there was no trend suggesting lower abnormal ICP rates in patients who had undergone lateral barrel staving, when compared to those who had not.

Does the addition of 3 or 4 lateral radial osteotomies, as in our series, fundamentally alter the nature of the 
wide-vertex sagittal suturectomy? Might one expect better outcomes with more extensive osteotomies? Although intuitively and to a certain extent historically, the answer to these questions has been "yes," there is little evidence in the literature to support this affirmation. Data regarding postoperative intracranial hypertension is scant, but most studies report morphological outcomes, as judged by the admittedly crude measure of the cephalic index. van Veelen and colleagues' recent study found no difference in the postsurgical cephalic index at 2 years between 4 different techniques of extended (modified) strip craniectomy. ${ }^{2}$ Indeed, when the results of all available studies are taken into account (summarized in the 2014 review of Bonfield and colleagues ${ }^{1}$ ), the morphological outcomes of extended strip craniectomy procedures and simple sagittal suturectomies are remarkably similar.

All extended, and indeed simple, sagittal strip craniectomies are passive procedures that depend upon the rapid expansion of the brain in the first year of life to drive the remodeling of the skull. Although the origin of raised ICP in sagittal craniosynostosis is not understood, one can certainly envisage a scenario in which over-rapid reossification of the calvarium, a reduced brain drive, or other physiological abnormality might result in inadequate cra- nial remodeling or intracranial volume in some individuals. This, in turn, could lead to elevated ICP later in life.

By contrast, calvarial remodeling (CR) performed after 12 months of age is an active procedure that restructures the calvarium overlying a brain with a slower growth velocity that is closer to its adult volume. It is probable that such an approach would be likely to have an overall lower incidence of subsequent craniocerebral disproportion than a passive surgical procedure conducted at an earlier age. This might well explain the differences we observed in the rate of raised ICP between patients undergoing MSC and CR. However, only future research that illuminates the true origin of raised ICP in these patients will finally answer this question.

\section{References}

1. Bonfield CM, Lee PS, Adamo MA, Pollack IF: Surgical treatment of sagittal synostosis by extended strip craniectomy: cranial index, nasofrontal angle, reoperation rate, and a review of the literature. J Craniomaxillofac Surg [epub ahead of print], 2014

2. van Veelen ML, Eelkman Rooda OH, de Jong T, Dammers R, van Adrichem LN, Mathijssen IM: Results of early surgery for sagittal suture synostosis: long-term follow-up and the occurrence of raised intracranial pressure. Childs Nerv Syst 29:997-1005, 2013 